\title{
EL NEMATODO BARRENADOR (Radopholus similis [COBB] THORNE) DEL BANANO Y PLÁTANO
}

\author{
ÓSCAR ADRIÁN GUZMÁN PIEDRAHITA ${ }^{1}$ \\ oscar.guzman@ucaldas.edu.co
}

Manizales, 2011-06-20 (Rev. 2011-10-04)

\section{RESUMEN}

Radopholus similis es un nematodo fitoparásito que se alimenta de raíces y cormos de banano y plátano en todo el mundo, afectando el crecimiento y desarrollo de este cultivo, con pérdidas en producción entre el 20 y $100 \%$. Debido a que estas musáceas han sido tradicionalmente propagadas por semilla asexual mediante colinos "cormos" o cepas "rizomas" y a que este fitonematodo se caracteriza por ingresar y movilizarse dentro de las células de raíces y los cormos, esto ha permitido que el intercambio de material de siembra infectado sea el principal medio de su diseminación alrededor del mundo. Por tales motivos, se describe a $R$. similis registrando su distribución, biología, pérdidas, hospedantes, supervivencia, medios de diseminación y prácticas de manejo como evitar el ingreso de los nematodos en el suelo antes de establecer un cultivo, reducir la cantidad de ellos en el material de siembra, promover la sanidad de las raíces de las plantas para ayudarlas a tolerar o competir con la presión de los nematodos u otros patógenos y reducir la oportunidad de que el nematodo ingrese a las raíces o rizomas.

\section{PALABRAS CLAVE:}

Fitoparásito, cormos, pérdidas, musáceas.

\author{
THE BANANA AND PLANTAIN BORER NEMATODE (Radopholus \\ similis [COBB] THORNE)
}

\section{ABSTRACT}

Radopholus similis is a phytoparasitic nematode which feeds itself from banana and plantain roots and corms worldwide, affecting the crops' growth and development with a loss in production between 20 and $100 \%$. Because these musaceae has been traditionally spread by asexual seeds through young coffee trees "corms" or "rhizome" stumps, and because this phytonemtode is characterized because it penetrates and moves into the roots' cells and the corms, this has allowed the exchange of infected sowing material to be the main means for its dissemination around the world. Because of this, $R$. similis is described registering its distribution, biology, losses, hosting, survival, dissemination means and management practices such as avoiding the penetration of nematodes in the soil before planting, reducing their quantity in the sowing material, promoting sanity of the roots in order to help them tolerate or compete with the nematodes or any other pathogen pressure, and reducing the probabilities for the nematode to penetrate roots and rhizomes.

\section{KEYWORDS}

Phytoparasitic, corms, losses, musaceae 


\section{INTRODUCCIÓN}

En los cultivos de banano y plátano, después de las lesiones foliares ocasionadas por las sigatokas negra y amarilla (Mycosphaerella fijiensis y M. musicola, respectivamente), los nematodos son los principales determinantes en afectar su crecimiento y desarrollo en el mundo debido al daño ocasionado en raíces y cormos (Robinson, Daneel \& Schoeman, 1998; Araya, 2003). Los nematodos son animales filiformes con cuerpo sin segmentos y más o menos transparentes, cubiertos de una cutícula hialina, que está marcada por estrías u otras marcas, son redondeados en sección transversal, con boca, sin extremidades u otros apéndices, muchos son parecidos a lombrices o con forma de anguila. Las hembras de algunas especies cuando llegan al estado adulto son abultadas con forma de pera o esfera (Siddiqi, 2000; Agrios, 2005; Perry \& Moens, 2006).

Así mismo, según el género de nematodo fitoparásito, tienen en la región anterior (cabeza) un órgano de alimentación llamado estilete hueco "lanza" (estomatoestilete u odontoestilete), que en algunas especies puede ser modificado (onquioestilete). El estilete es usado para perforar o penetrar las células de las raíces y el cormo u otros órganos como tallos, hojas y frutos, y a través de él extraen los nutrientes de la plantas hospedantes, causando enfermedades en diferentes cultivos que se manifiestan con un crecimiento deficiente y un menor rendimiento (Maggenti, Luc, Raski, Fortuner \& Geraert, 1987; Mai, Mullin, Lyon \& Loeffler,1996; Montiel, Sosa, Medrano \& Romero, 1997; Agrios, 2005; Luc, Sikora \& Bridge, 2005; Perry \& Moens, 2006).

En plantaciones con varios años de establecidas, es común encontrar comunidades poliespecíficas, compuestas por endoparásitos migratorios como Radopholus similis (Cobb) Thorne y Pratylenchus coffeae Sher \& Allen, los ecto-endoparásitos Helicotylenchus multicinctus Cobb y $\mathrm{H}$. dihysteria Cobb, los endoparásitos sedentarios Meloidogyne incognita (Kofoid y White) Chitwood y Meloidogyne javanica (Treub) Chitwood y el semi-endoparásito Rotylenchulus reniformis Lindford y Oliveira (Araya, 2003, 2004; Gowen, Quénéhervé \& Fogain, 2005; Martínez, Pena \& Espinal, 2006). En adición a estas cinco especies, hay muchas otras que han sido registradas en Musa spp., a través del mundo (Gowe et al., 2005). Sin embargo, la frecuencia y abundancia de cada una de estas especies puede cambiar según sea la culti-variedad de banano o plátano y las condiciones agroecológicas (Araya, 2003).

De acuerdo con las frecuencias y densidades poblacionales, durante el año y en las zonas productoras de plátano y banano de cada país, $R$. similis es el más abundante y la principal especie fitoparásita, constituyendo entre el 82 y $97 \%$ de la población de nematodos en raíces y cormos (Araya \& Moens, 2003); y cuando este fitonematodo no es controlado, se disminuye el peso de los racimos $y$, por efecto del volcamiento, se reduce el rendimiento $\left(\mathrm{t} \mathrm{ha}^{-1}\right)$ entre 60 y $52 \%$ en la primera y segunda cosecha, respectivamente (Fogain, 2000), pero la reducción en el rendimiento puede llegar hasta un $80 \%$ (Figura 1) (Moens, Araya, Swennen \& De Waele, 2004).

Debido a que el banano y el plátano han sido tradicionalmente propagados por semilla asexual mediante colinos "cormos" o cepas "rizomas" (Aguas \& Martínez, 2003), y a que R. similis se caracteriza por ingresar y movilizarse dentro de las células de raíces y los cormos, esto ha permitido el intercambio de material de siembra infectado sea el principal medio de su diseminación (Loos, 1962; Blake, 1969; Sarah, Pinochet \& Stanton, 1996; Sarah, 2000; Gowen et al., 2005). Como muchas especies de plantas son transportadas por los seres humanos, en especial las de propagación asexual, los parásitos y patógenos son 
también movidos con ellos, como es el caso de R. similis en estas musáceas (Sarah et al., 1996; Marín, Sutton \& Barker, 1998).

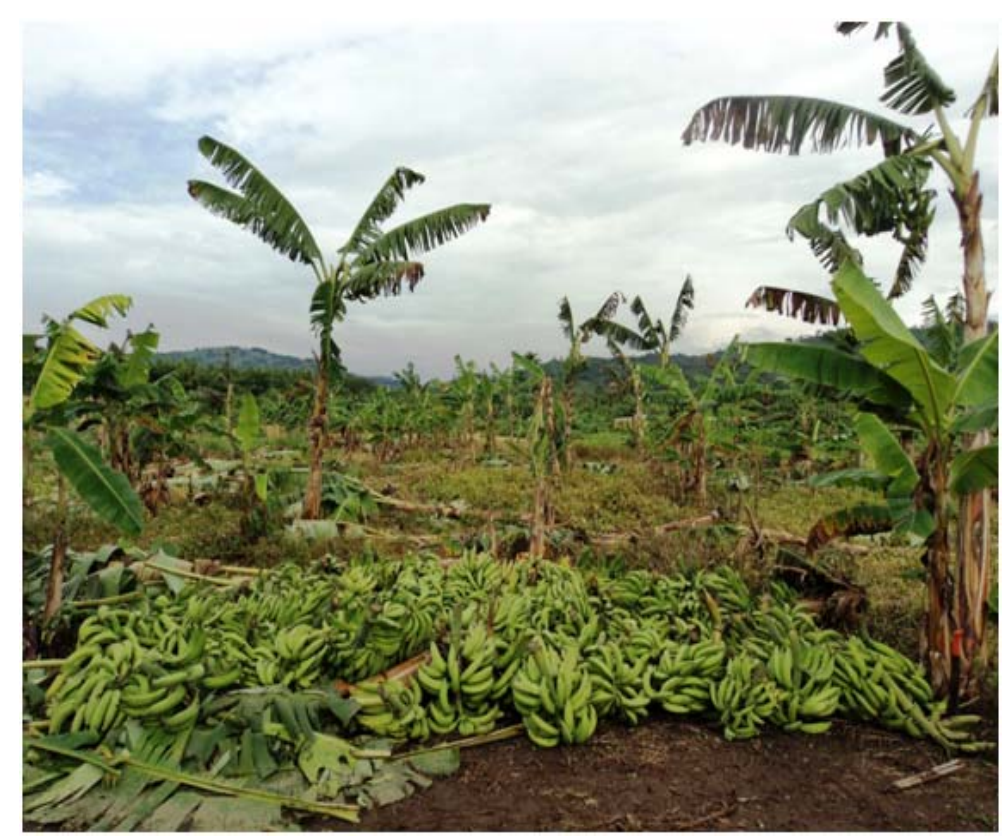

Figura 1. Cultivo de plátano Dominico Hartón en la Granja Montelindo de la Universidad de Caldas (Palestina, Caldas, Colombia), severamente afectado por el nematodo barrenador $R$. similis. En el fondo se observan plantas volcadas, y al frente racimos recolectados de plantas caídas $\sin$ completar su desarrollo.

\section{EL NEMATODO BARRENADOR (Radopholus similis)}

\section{A) Primer registro}

R. similis fue observado por primera vez por Nathan August Cobb (el padre de la nematología de Occidente) en julio de 1891 en Nueva Gales del Sur, en raíces necróticas de Musa sapientum, provenientes de las islas Fiji. Dos años después (1892), Cobb nombró al nematodo como Tylenchus similis y registró que las hembras no fueron observadas (Cobb, 1892). Posteriormente, en 1915, Cobb recibió cormos de banano Gros Michel infectados con este nematodo enviados desde Jamaica y encontró especímenes adicionales, que fueron usados para realizar una descripción y publicación más completa de la especie (Cobb, 1915; Thorne, 1961).

\section{B) Distribución}

Después del primer registro, $R$. similis ha sido encontrado en las regiones tropicales y subtropicales donde crece banano y plátano, excepto en Israel, Islas Canarias, Islas Cabo Verde, Chipre, Creta, Mauricio y Taiwán (Gowen et al., 2005). A pesar de que Radopholus es un género originario de Australia y Nueva Zelanda, la especie $R$. similis se encuentra en las áreas tropicales y subtropicales del mundo (Thorne, 1961; Sher, 1968; Román, 1978; Araya, 2003). 


\section{C) Nombre de la enfermedad}

En el idioma inglés, la enfermedad ocasionada por $R$. similis en plátano y banano es conocida como "Burrowing nematode disease, Nematode root rot, Toppling disease, Blackhead disease o Black head toppling disease" (Gowen et al., 2005; Brooks, 2008; APS, 2011); en español se conoce con los nombres de "Cabeza negra o volcamiento" (Figura 2) (Román, 1978) y Arranque (Grisales \& Lescot, 1999).
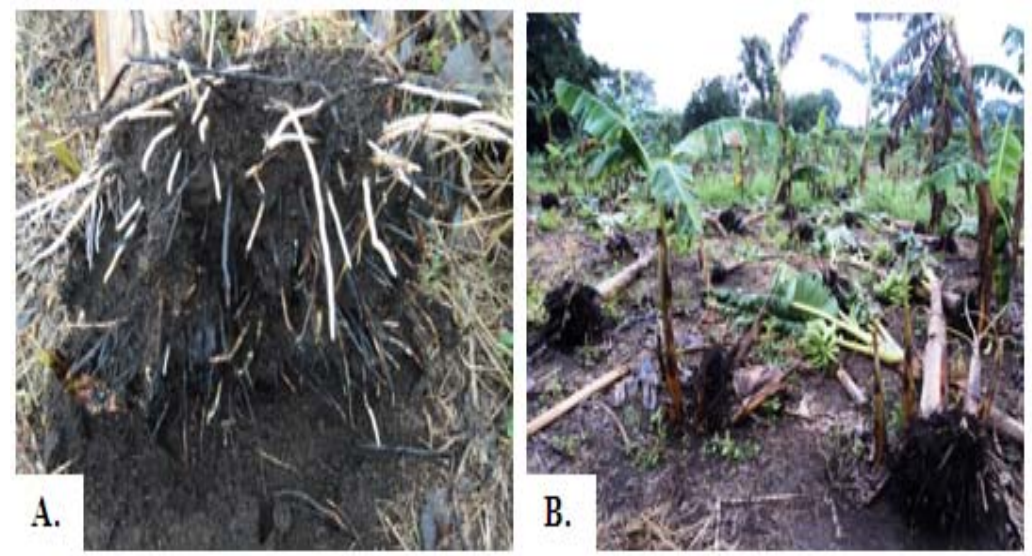

Figura 2. A. Enfermedad Cabeza negra en plátano Dominico Hartón. B. Volcamiento de plantas ocasionado por $R$. similis.

\section{D) Ubicación taxonómica}

Según Thorne (1961) y Siddiqi (2000), R. similis pertenece al Phyllum Nematoda Rudolphi, 1808, Clase Secernentea (von Linstow, 1905) Dougherty, 1958, Orden Tylenchida Thorne 1949, Superfamilia Tylenchoidea Oerley, 1880, Familia Tylenchidae Oerley, 1880 y Subfamilia Pratylenchinae Thorne, 1949.

\section{E) Biología}

De acuerdo a Blake (1961), Loos (1962), Blake (1966), Román (1978), Kaplan (1994), Sarah et al. (1996), Marín et al. (1998), Sarah (2000), Siddiqi (2000), Nguyet et al. (2003), Araya (2004), Gowen et al. (2005) y Brooks (2008), las principales características del ciclo de vida (Figura 3) y parasitismo de $R$. similis son:

\section{Ciclo de vida}

1. Como la mayoría de nematodos, los estados de desarrollo de R. similis son vermiformes o con forma de lombriz y tienen cuatro estados juveniles (J1, J2, J3 y J4) y el adulto. El estado J1 se desarrolla dentro del huevo, luego muda la cutícula y luego de 8 a 10 días emerge el J2. Los estados J2, J3 y J4 también mudan la cutícula hasta llegar al estado adulto entre 10 y 13 días.

2. El estado J2 y la hembra adulta son infectivos y tienen formas móviles que pueden abandonar las raíces en condiciones adversas, llegando al suelo para parasitar nuevamente raíces sanas. El movimiento de los juveniles y las hembras es estimulado por factores nutricionales, ya que necesitan tejido sano para alimentarse. Después de iniciar su alimentación, R. 
similis completa su ciclo de vida entre 20 y 25 días en los tejidos de las raíces y cormos a una temperatura entre 24 y $32^{\circ} \mathrm{C}$; siendo óptima su reproducción entre 25 y $28^{\circ} \mathrm{C}$.

3. Los machos de R. similis se diferencian de las hembras que son morfológicamente diferentes a ellos, teniendo dimorfismo sexual por caracteres secundarios; esto significa que los machos son diferentes a las hembras especialmente en la región anterior (cabeza) que es elevada (sobresaliente), y allí exhiben un estilete poco desarrollado con perillas basales apenas visibles, debido a esto se cree que no son fitoparásitos (Figura 3). En la región posterior (cola), los machos poseen una espícula de función reproductiva, cubierta por una membrana hialina llamada bursa (Figura 3).

4. Las hembras miden entre 500 y $880 \mu \mathrm{m}(0,50$ y $0,88 \mathrm{~mm})$ y cerca de $24 \mu \mathrm{m}$ de diámetro, con un estilete bien desarrollado de 16 a $21 \mu \mathrm{m}$ de largo (promedio de $18 \mu \mathrm{m}$ ). Usualmente se reproducen sexualmente, pero pueden reproducirse sin los machos utilizando estrategias partenogenéticas. Dentro del tejido infectado, las hembras colocan cuatro o cinco huevos por día durante 2 semanas (Figura 3).

5. Por contraste, los machos son más pequeños que las hembras, miden 500 a $700 \mu \mathrm{m}(0,50$ y $0,70 \mathrm{~mm})$. Los estados juveniles miden entre 315 a $400 \mu \mathrm{m}$ y exhiben un estilete de aproximadamente 13 a $14 \mu \mathrm{m}$ de longitud.

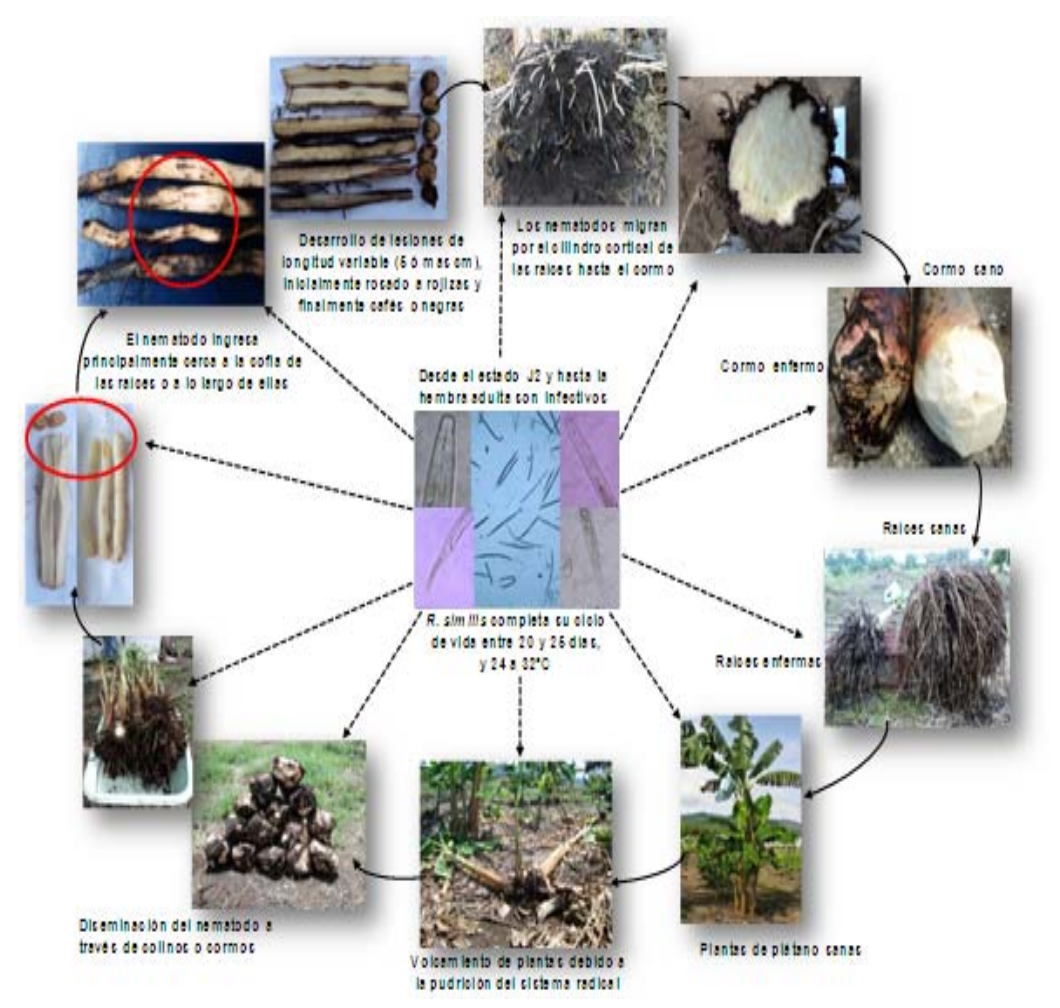

Figura 3. Ciclo biológico y patológico del nematodo barrenador Radopholus similis.

\section{Parasitismo}

1. R. similis es un parásito obligado de tejidos de plantas, es decir, necesita de un hospedante vivo para sobrevivir (Siddiqi, 2000; Brooks, 2008). En el tejido radicular y los cormos, allí vive como endoparásito migratorio (Figuras 4A, B, C y D) y cormos (Figuras 
4E, F, G y H), y realiza completamente su ciclo de vida (Blake, 1961; Loos, 1962; Sarah et al., 1996; Sarah, 2000).

2. El nematodo perfora la pared celular e ingresa principalmente cerca a la cofia de las raíces, o lo largo de ellas, mediante su estomato-estilete y luego se alimenta del citoplasma (Figuras 4A, $B, C$ y D). Tanto juveniles como adultos viven en el parénquima cortical, donde se mueven activamente causando daño conforme se alimentan del citoplasma de las células vecinas.

3. Las células son destruidas y el nematodo migra intra e intercelularmente en el cilindro cortical (Figuras 4C y D), y los cormos (Figuras 4G y $\mathrm{H}$ ); en el primero, hace cavidades o lesiones que cuando hay altas infestaciones, las lesiones en la raíz coalesen, anillándola completamente (Figuras 4C y D). Estas cavidades, semejan agujeros barrenados en las células, bajo esta condición se derivó el nombre de nematodo barrenador.

\section{F) Síntomas primarios}

En las células del cilindro cortical, $R$. similis produce lesiones de longitud variable (5 o más $\mathrm{cm}$ ) con forma de estrías, éstas inicialmente tienen colores que varían desde amarillo claro hasta oscuro (Figura 4A), luego rosado rojizas (Figura 4B) y finalmente marrón o negras. En algunos casos produce depresiones en el tejido que modifican la anatomía cilíndrica original de las raíces (Figuras 4C y D) (Blake, 1961; Thorne, 1961; Blake, 1966; Fogain \& Gowen, 1997; Valette, Andary, Geiger, Sarah \& Nicole, 1998; Oramas \& Román, 2006). En infestaciones altas, las lesiones rodean completamente las raíces, hasta destruirlas totalmente (Figura 4D) (Sarah et al., 1996; Marín et al., 1998; Araya \& De Waele, 2004; Gowen et al., 2005). Dicha coloración se caracteriza por estar infestada con todos los estados de desarrollo del nematodo (Sarah et al., 1996; Marín et al., 1998; Gowen et al., 2005). Blake (1966) y Oramas y Román (2006), encontraron que $R$. similis no solo causa daño físico, sino que también causa daño fisiológico al producir hipertrofia del núcleo y nucléolo de las células.

En las raíces, $R$. similis no invade el cilindro central (tejidos vasculares) (Figuras 4H e I), aun en infestaciones altas (Blake, 1961, 1966; Thorne, 1961; Oramas \& Román, 2006), el cual queda expuesto a la invasión de microorganismos secundarios como hongos y bacterias, siendo los más comunes Cylindrocarpon musae, Acremonium stromaticum y Fusarium spp., que ocasionan necrosis y muerte de las raíces en la parte posterior de la lesión (Figuras 4H e I) y pueden contribuir al volcamiento (Stover, 1972; Sarah et al., 1996; Sarah, 2000; Marín et al., 1998; Araya, 2003; et al., 2005). El nematodo puede migrar de las raíces al cormo (rizoma) causando lesiones de hasta $6 \mathrm{~cm}$ de profundidad (Figuras 4G, H e I). 

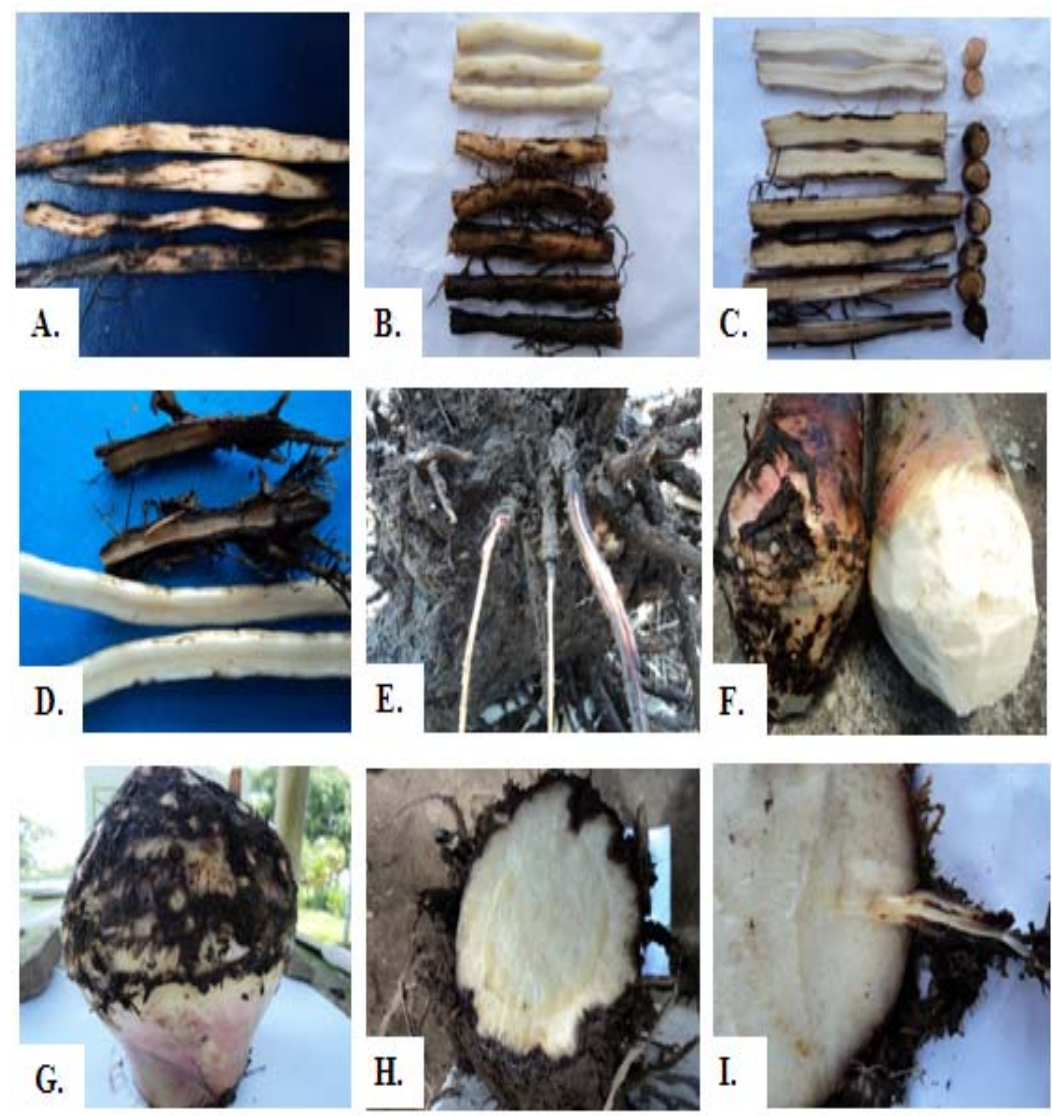

Figura 4. A. Diferentes sitios de penetración de $R$. similis en raíces. B-C. Destrucción de células del cilindro cortical por el nematodo barrenador. D. Cilindro cortical y central destruidos por el ataque del nematodo y microorganismos secundarios (arriba), y tejido sano (abajo). E. Cilindro central sano (color blanco) y cilindro cortical enfermo (color marrón y negro). F. Cormo parasitado por el nematodo (izquierda) y sin parasitar (derecha). G. Cormo y primordios de raíces parasitados. H-I. Corte transversal de cormos donde se aprecia la necrosis externa producida por el nematodo.

\section{G) Síntomas secundarios}

En las plantas infectadas por $R$. similis se reduce la absorción de agua y nutrientes, resultando en varios síntomas como amarillamiento de hojas y disminución del tamaño y longevidad de las plantas (Figura 5A, B), los cuales pueden ser fácilmente confundidos con deficiencias nutricionales. Los colinos infectados por $R$. similis son de menor tamaño y vigor, con hojas más pequeñas $y$, en algunos casos, sobresalen del suelo (enbalconados) y con el peciolo de las hojas viejas necrótico (Figura $5 \mathrm{C}$ ), diferente a los colinos de las plantas sanas (Figura 5D). Las diferencias en los síntomas, son también determinadas por las características químicas y físicas del suelo, disponibilidad de nutrientes, la especie hospedante y el género de fitonematodo involucrado (Thorne, 1961; Jenkins \& Taylor, 1967; Sarah et al., 1996). Lo anterior, se refleja en un menor número y tamaño de las hojas y menor peso del racimo, en un incremento del tiempo de siembra a floración, de floración a cosecha, entre floraciones y entre cosechas (Sarah et al., 1996; Araya, 2003; Gowen et al., 2005). 
Finalmente, las plantas pierden anclaje por el deterioro del sistema radical, por lo cual tienden a desraizarse o volcarse, esto puede ocurrir en plantas jóvenes (Figura 5E), y adultas (Figura 5F), principalmente entre la época de floración y cosecha debido al peso del racimo, particularmente durante vientos y lluvias fuertes, lo que causa pérdidas económicas altas (Loos \& Loos, 1960a; Sarah et al., 1996; Montiel et al., 1997; Sarah, 2000; Araya, 2003; Brooks, 2008).

En plantas de la misma edad, se puede apreciar que las severamente atacadas por $R$. similis carecen de raíces absorbentes y las raíces de anclaje son necróticas, de menor tamaño y cantidad, contrario a lo que ocurre en el sistema radical de una planta que no es atacada por este fitonematodo (Figura 5G) (Loos \& Loos, 1960a; Araya, 2003).
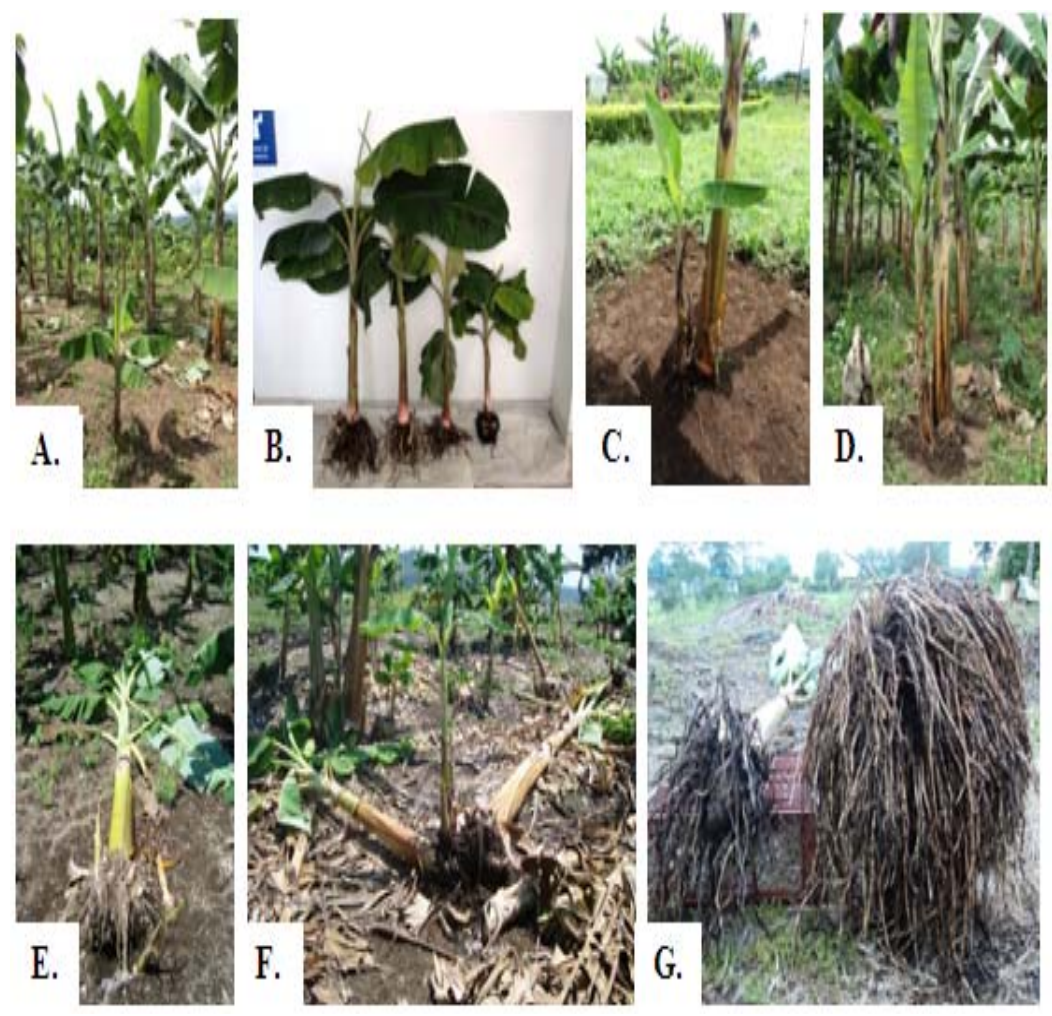

Figura 5. A. Plantas de Dominico Hartón infectadas por R. similis con síntomas como amarillamiento de hojas y disminución del tamaño. B. Plántulas de Dominico Hartón de la misma edad, sanas (izquierda) y enfermas por R. similis (derecha). C. Colinos con menor crecimiento en plantas parasitadas. D. Colinos con mayor desarrollo en plantas sanas. EF. Plantas joven y adulta volcadas debido a la destrucción de raíces por $R$. similis, respectivamente. G. Plantas de la misma edad, severamente atacada por $R$. similis (izquierda) y sin atacar (derecha).

\section{H) Pérdidas}

Las pérdidas en la producción se estiman en un $20 \%$ (Sasser \& Freckman, 1987). Según Araya (2004), evaluaciones precisas de los efectos de los nematodos en la producción de banano son escasas, pero algunas estimaciones que se reportan en la literatura indican que las pérdidas pueden llegar hasta el $100 \%$, las cuales, dependen del cultivar, el tipo de suelo y las condiciones agroecológicas. En plantaciones infectadas con control deficiente, las pérdidas en rendimiento pueden estar entre un 44 y 50\% (Loos \& Loos, 1960b; Araya, 2003). En 
condiciones extremas, en suelos pobres y erosionados, las pérdidas acumulativas durante tres ciclos de producción pueden alcanzar hasta $75 \%$, debido a la reducción del peso del racimo y a la caída de las plantas como ha ocurrido en Costa de Marfil (Sarah et al., 1996). Así mismo, el autor de éste artículo ha encontrado pérdidas del $70 \%$ ocasionadas por $R$. similis, principalmente por efecto del volcamiento, en plátano Dominico Hartón en la Granja Montelindo de la Universidad de Caldas (Colombia), en el primer ciclo de producción.

Fogain (2000), encontró un mayor número de plantas caídas (18,3 y $52,5 \%$, en la primera y segunda cosecha, respectivamente) en áreas infestadas por $R$. similis sin tratar con nematicida, y un menor número de plantas caídas $(2,5$ y $7,5 \%$, en la primera y segunda cosecha, respectivamente) en las áreas tratadas con nematicidas. Lo anterior, disminuyó el rendimiento (t ha-1) en un 60 y $52 \%$ en las plantas enfermas, en la primera y segunda cosecha, respectivamente, en comparación con las plantas tratadas. Estos resultados muestran cómo el efecto es gradual y acumulativo, debilitando la unidad de producción, la cual pierde vigor, longevidad y dependiendo de las condiciones de la plantación en tres o cuatro generaciones los racimos que se producen difícilmente llegan a satisfacer los requerimientos de exportación (Araya, 2004). La respuesta a los nematicidas varía con las condiciones físico-químicas del suelo, condiciones climáticas, el cultivar y el nematodo que se esté controlando. Hay aumentos hasta de un $376 \%$, pero pueden ser tan variables como desde un $5 \%$ hasta $267 \%$ (Araya, 2003).

En América Central (Costa Rica y Panamá) y en América del Sur (Colombia y Ecuador), las pérdidas causadas por el volcamiento de plantas fluctúan entre 12 y $18 \%$, mientras que en el Valle de Sula en Honduras, éstas tienden a ser menores, alrededor del 5\% (Araya, 2003). En plantas de Grande Naine, cultivar de banano Cavendish, $R$. similis redujo el peso de las raíces en un $66 \%$, mientras que el daño ocasionado a las raíces fue más alto y el peso de los racimos más bajo, en plantas inoculadas con $R$. similis y $P$. coffeae, en comparación con el testigo (Moens et al., 2004).

Moens, Araya, Swennen, De Waele \& Sandoval (2003), cuando inocularon $R$. similis con incrementos de densidades desde 0,14 a 2,24 nematodos $\mathrm{ml}^{-1}$ de sustrato, correspondiendo a 254 y $2128 R$. similis por vaso de 1,8 $\mathrm{L}$, respectivamente, encontraron una reducción lineal del peso de las raíces. En ellas, por cada 1.000 nematodos inoculados se redujo el peso en $3,9 \mathrm{~g}(16 \%)$. Así mismo, Fallas, Sarah \& Fargette (1995), inocularon $100 R$. similis en plantas de Valery en vasos de 0,8 L, y encontraron una disminución en el peso de raíces entre 11 a $53 \%$ después de 12 semanas.

\section{I) Hospedantes}

La mayoría de materiales de banano y plátano de las variedades comestibles de Musa AA, AAA, AB, AAB y ABB son atacados por $R$. similis (Wehunt, Hutchinson \& Edwards, 1978; Davide \& Marasigan, 1985), también como abacá (Gowen et al., 2005). Los cultivares Grande Naine, Valery y Williams del Subgrupo Cavendish que son cultivados en Latinoamérica son susceptibles a R. similis, Helicotylenchus multicinctus, $H$. dyhisteria, Meloidogyne incognita, M. javania y Pratylenchus coffeae (Araya, 2004).

En las Américas, $R$. similis parece estar confinado a Musa spp., y a algunas plantas cultivadas Piper nigrum, Saccharum spp., Citrus spp., Coffeea spp. y ornamentales como Anthurium andraeanum (Román, 1978; Quénéhervé et al., 2005). Brooks (2008), reportó más de 350 especies de plantas que son susceptibles a Radopholus spp., en las que se incluyen 
banano, cítricos, pimienta negra, antúrios, aráceas (antúrios, taro), jengibre, té, coco y otras palmas.

En la Granja Montelindo de la Universidad de Caldas, departamento de Caldas (Colombia), $R$. similis ha sido encontrado en 13 especies de arvenses principalmente en Borreria laevis (Rubiaceae) (Figura 6A), Phyllanthus corcovadensis (Euphorbiaceae) (Figura 6B), Lantana cámara (Verbenaceae) (Figura 6C) y Sida acuta (Malvaceae) (Figura 6D) (Rivera, Guzmán \& Zamorano, 2011); así mismo, este fitonematodo es encontrado en malezas de cultivos de banano en Martinica, principalmente especies de las familias Poaceae, Euphorbiacae y Solanaceae (Quénéhervé, De Bock, Valette \& Chabrier, 2000).
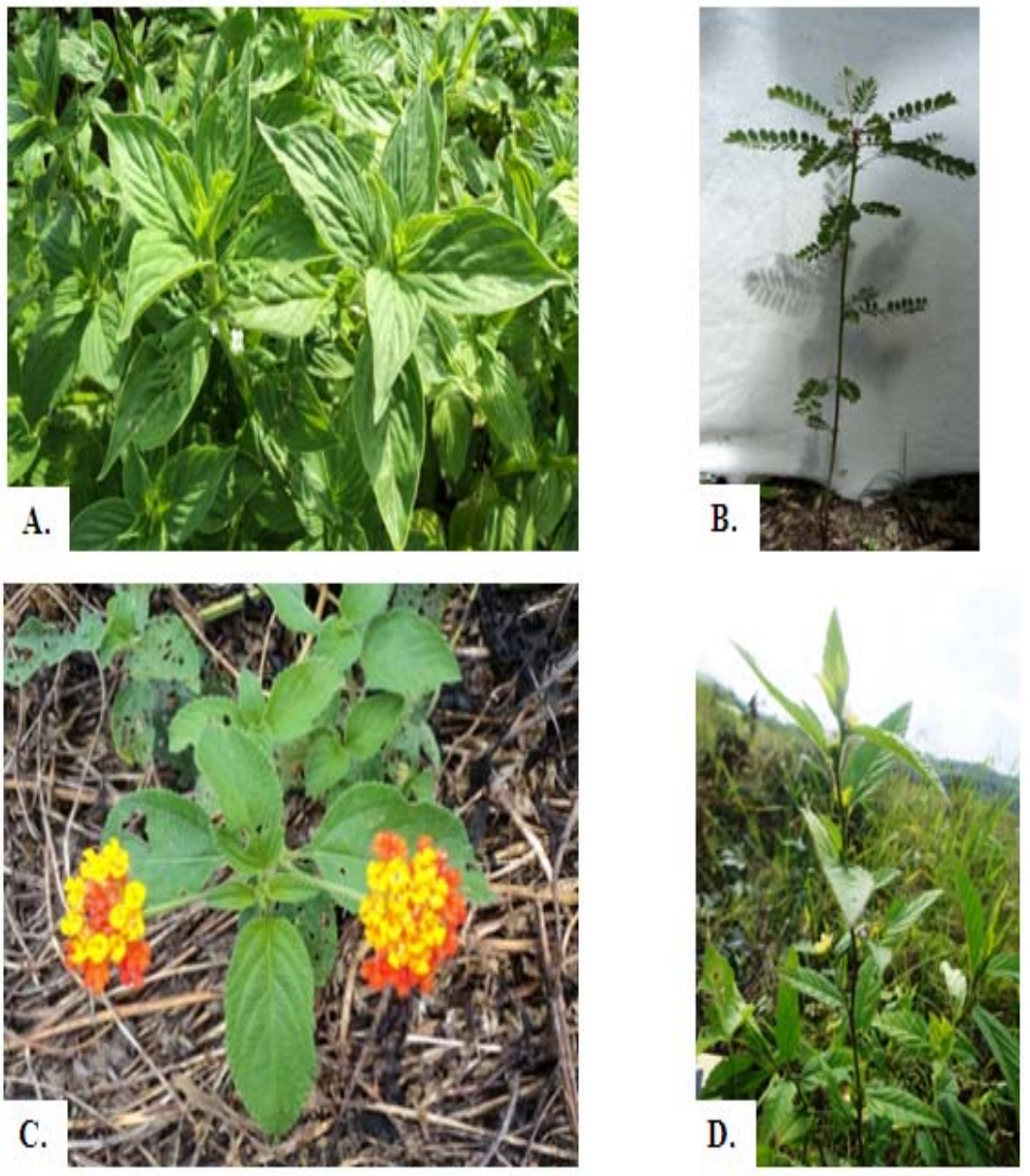

Figura 6. Principales arvenses hospedantes de $R$. similis en la Granja Montelindo de la Universidad de Caldas (Colombia). A. Borreria laevis. B. Phyllanthus corcovadensis. C. Lantana cámara. D. Sida acuta.

\section{J) Supervivencia}

$R$. similis tiene una vida corta en el suelo, de seis meses o menos. Como todos los organismos biotróficos depende de hospedantes vivos, como los antes mencionados, para sobrevivir.

- En tejidos vegetales: $R$. similis sobrevive hasta seis meses dentro de raíces infectadas, cormos y arvenses hospedantes (Gowen et al., 2005). Tarjan (1961) y Loos (1962) demostraron que $R$. 
similis no sobrevive en el suelo por más de seis meses en la ausencia de raíces hospedantes o trozos de cormos vivos.

- En el suelo: aunque las poblaciones en el suelo son por lo general bajas, los huevos, estados juveniles y adultos de $R$. similis pueden sobrevivir seis meses allí y ser transportados (Gowen et al., 2005).

\section{K) Medios de diseminación}

La dispersión pasiva de los nematodos en agua de escorrentía y a través de sistemas de riego, es potencialmente grave para los agricultores tratando de eliminar a $R$. similis en las zonas donde los campos infestados de banano y plátano están adyacentes a las nuevas plantaciones.

Como el nematodo sobrevive en raíces y cormos, es por esto que el material de siembra es el principal medio de diseminación entre lotes, regiones o países. Así mismo, a través del suelo infestado, herramientas, maquinaria, botas, etc., también se puede diseminar el nematodo (Gowen et al., 2005).

La amplia distribución de $R$. similis en América latina y el Caribe, parece estar correlacionada con las áreas donde las plantas de banano del Subgrupo Cavendish (AAA) fueron importadas. Se especula que en América Latina y el Caribe, $R$. similis fue introducido en banano en la variedad Gros Michel que posteriormente infectó los cultivares Cavendish que son más susceptibles (Marín et al., 1998; Gowen et al., 2005).

\section{L) Principales prácticas de manejo de $R$. similis}

Las alternativas de manejo integrado de $R$. similis están determinadas por varios factores agronómicos que se mencionarán a continuación, según los trabajos realizados por: Loos \& Loos (1960b); Blake (1961); Tarjan (1961); Loos (1962); Stover (1972); Sieverding (1991); Guerrero (1996); Sarah et al. (1996); Smith \& Read (1997); Daneel, De Jager \& De Beer (1998); Green \& Afreh-Nuamah (1998); Mbwana \& Rukazambuga (1998); Okech, Karamura \& Gold (1998); Robinson et al. (1998); Seshu et al. (1998); Sarah (2000); Guzmán \& Castaño (2002); Araya (2003); Hauser (2003); Gowen et al. (2005); Price (2006) y Ramírez \& Castaño (2009).

1) Evitar el ingreso de los nematodos en el suelo antes de establecer un cultivo, mediante las siguientes prácticas:

- Debido a que R. similis no se encuentra en suelos no infestados, sin vocación agrícola o vírgenes, se debe evitar su ingreso a ellos antes de la siembra. Para lograr esto, es esencial utilizar material de siembra limpio libre de fitonematodos para establecer la nueva plantación.

- Realizar análisis de nematodos fitoparásitos en raíces y suelo para conocer la presencia o ausencia de otros géneros y sus poblaciones.

- Tratar de hacer solarización, es decir, tratamiento del suelo con calor por radiación solar.

2) Reducir la cantidad de nematodos en el material de siembra, mediante las siguientes prácticas:

- Cuando sea posible, utilizar plantas provenientes de cultivos de tejidos (in vitro). 
- Obtención de colinos de plantas libres de nematodos fitoparásitos y otros agentes causantes de enfermedades.

- Como R. similis sobrevive en cormos y raíces infectadas, realizar remoción de capas externas del rizoma "pelado" de tejido infectado para disminuir su población; además, esta práctica permite hacer inspección del tejido blanco para observar la presencia de picudos.

- La práctica de la limpieza sanitaria "pelado" de los cormos se debe realizar fuera del campo, y los cormos necróticos severamente lesionados deben ser descartados y dejar solamente los limpios para la siembra. Esta actividad es relativamente fácil y no es costosa.

- Eventualmente, se puede hacer inmersión de colinos en agua caliente a $55^{\circ} \mathrm{C}$ por 20 min o en agua hirviendo por $1 \mathrm{~min}$.

- Inmersión de cormos en solución de aldicarb en dosis de $10 \mathrm{~mm} \mathrm{~L}$ 1 , y carbofuran dosis de $20 \mathrm{ml} \mathrm{L}^{-1}$ durante $60 \mathrm{~min}$.

3) Promover la sanidad y el vigor de las raíces de las plantas para ayudarlas a tolerar o competir con la presión de los nematodos u otros patógenos, mediante las siguientes prácticas:

- Aplicación de nematicida en el sitio de siembra y en suelo. En cultivos comerciales, cuando las poblaciones de nematodos son altas, la manera más común de controlarlo es con la aplicación frecuente de productos químicos organofosforados o carbamatos (no volátiles), los cuales son aplicados como gránulos sobre la superficie del suelo alrededor de la planta.

- Preparación óptima del suelo antes de la siembra, ya que en suelos sueltos ocurre mejor crecimiento y desarrollo del sistema radical, reflejado en una mayor cantidad y longitud de raíces, contrario a lo que sucede en suelos pesados y compactados.

- Incorporación constante de enmiendas orgánicas bien procesadas como gallinaza, pulpa de café, cenichaza, porcinaza, etc., las cuales promueven la sanidad y el vigor de las raíces en cultivos de musáceas, permitiéndoles competir mejor con los nematodos. Los principales beneficios son: 1) incremento y reposición de la materia orgánica del suelo, 2) reducción de la temperatura de la superficie y las fluctuaciones de temperatura, 3) reducción del crecimiento de arvenses, 4) mejoramiento de la estructura del suelo e infiltración de agua, 5) disminución de la erosión del suelo por el viento (menos polvo), 6) disminución de la erosión por la escorrentía, 7) reducción de la compactación del suelo, 8) disminución de la pérdida de agua por evaporación de la superficie, 9) mayor crecimiento y vigor de las raíces, y 10) estimulación de la microflora y microfauna del suelo, la cual indirectamente reduce los fitonematodos.

- Realizar fertilización química de las plantas de acuerdo con requerimientos nutricionales y análisis de suelo.

4) Reducir la oportunidad de que el nematodo ingrese a las raíces o rizomas, mediante el uso de insumos biológicos como:

- Aplicación en mezcla con agua de hongos y bacterias biorreguladores como Paecilomyces lilacinus y Pasteuria penetrans, que ayudan a controlar los fitonematodos.

- Incorporar insumos biológicos como las micorrizas arbusculares (MA), que son un grupo de hongos habitantes naturales del suelo que forman simbiosis con las raíces de las plantas para favorecer la absorción y transporte de macro y micro-nutrimentos, especialmente los poco móviles como fósforo, zinc, cobre, lo cual 
conlleva a una menor utilización de fertilizantes químicos y a un mayor crecimiento de las plantas. Las MA presentan otros beneficios a las plantas como una mayor capacidad de absorción de agua y tolerancia a la sequía, protección de las raíces contra patógenos presentes en el suelo y detoxificación de metales pesados.

5) Otras prácticas de manejo:

- Dejar el lote donde se tenían musáceas sin sembrar por seis meses o más tiempo, hasta dos años, para que descanse (barbecho). Durante este período, los rizomas viejos, colinos y otros tejidos de plátano o banano deben ser removidos para eliminar los nematodos, ya que $R$. similis puede sobrevivir en ellos por seis meses; así mismo, se debe evitar el crecimiento de malezas u hospedantes alternos como los mencionados anteriormente.

- En cultivos con poblaciones altas de R. similis, realizar rotación con especies no hospedantes como yuca, por un mínimo de dos años.

- Evitar el volcamiento de plantas sosteniéndolas con postes de guadua u otro material. En algunas fincas se utiliza el amarrado de las plantas para evitar su caída.

- Proporcionar buen drenaje en zonas de lluvia intensa.

- Sembrar cultivares vigorosos y con tolerancia a vientos.

- Si la topografía del terreno es plana y existe disponibilidad de agua, realizar inundación por ocho semanas después de haber destruido previamente el cultivo de plátano y banano. Aunque esta práctica es poco común.

\section{BIBLIOGRAFÍA}

- Agrios, G. (2005). Plant pathology. 5 ed. Nueva York: Elsevier Academic Press. 922p.

- Aguas, A. A., y Martínez, M. (2003). Técnicas rápidas para la multiplicación de semillas de plátano. Boletines Divulgativos Ecorregión Caribe (COL), 69. 7p.

- American Phytopathological Society -APS-. (2011). Diseases of Banana and Plantain (Musa spp.). APSnet. Common Names of Plant Diseases. Obtenido el 7 de junio de 2011, desde http://www.apsnet.org/publications/commonnames/Pages/Banan aandPlantain.aspx

- Araya, M. (2003). Situación actual del manejo de nematodos en banano (Musa AAA) y plátano (Musa AAB) en el trópico americano. En Rivas, G., y Rosales, F. (eds.), Manejo Convencional y alternativo de la Sigatoka Negra, Nematodos y Otras Plagas Asociadas al Cultivo de Musáceas en los Trópicos (pp. 79-102). Francia: INIBAP.

- (2004). Los fitonematodos del banano (Musa AAA Subgrupo Cavendish cultivares Grande Naine, Valery y Williams) su parasitismo y combate. XVI Reunión Internacional ACORBAT. pp. 84-105.

- Araya, M., y De Waele, D. (2004). Spatial distribution of nematodes in three banana (Musa AAA) root parts considering two root thickness in three farm management systems. Acta Oecologica, 26, 137-148.

- Araya, M., y Moens, T. (2003). Parasitic nematodes on Musa AAA (Cavendish subgroup cvs 'Grande naine' 'Valery' and 'Williams'). 
En Turner, D., y Rosales, F. (eds.), Banana Root System: Toward a Better Understanding for Its Productive Management (pp. 201-223). San José, Costa Rica, 3-5 november. Montpellier, France: INIBAP.

- Blake, C. D. (1961). Root rot of bananas caused by Radopholus similis (Cobb) and its control in New South Wales. Nematologica, 6, 295-310.

- $\quad$ (1966). The histological changes in banana roots caused by Radopholus similis and Helicotylenchus multicinctus. Nematologica, 12, 129-137.

- (1969). Nematode parasites of banana and their control. En Peachy, J. E. (ed.), Nematodes of Tropical Crops (pp. 109132). Technical Communication No. 40. St Albans, UK: Commonwealth Bureau of Helminthology.

- Brooks, F. E. (2008). Burrowing Nematode. The Plant Health Instructor. APSnet. Obtenido el 4 de marzo de 2011, desde http://www.apsnet.org/edcenter/intropp/lessons/Nematodes/Pag es/Burrowingnematode.aspx

- Cobb, N. A. (1892). Banana disease in Fiji. Bull. Misc. Info., Royal Gardens, Kew (London). pp. 48-49.

- . (1915). Tylenchus similis, the cause of a root disease of sugar cane and banana. J. Agric. Res., 4, 561-68.

- Daneel, M., De Jager, K., y De Beer, Z. (1998). IPM for nematodes on bananas in South Africa. En Frison, E. A., Gold, C. S., Karamura, E. B., y Sikora, R. A. (eds.), Mobilizing IPM for Sustainable Banana Production in Africa (pp. 247-257). Proceedings of a Workshop on Banana IPM Held in Nelspruit, South Africa - 23-28 November 1998. INIBAP.

- Davide, R. G., y Marasigan, L. Q. (1985). Yield loss assessment and evaluation of resistance of banana cultivars to the nematode Radopholus similis Thorne and Meloidogyne incognita Chitwood. Phil. Agri., 68, 335-349.

- Fallas, G. A., Sarah, J. L., y Fargette, M. (1995). Reproductive fitness and pathogenicity of eight Radopholus similis isolates on banana plants (Musa AAA cv. Poyo). Nematropica, 25(2), 135141.

- Fogain, R. (2000). Effect of Radopholus similis on plant growth and yield of plantain (Musa AAB). Nematology, 2, 129-133.

- Fogain, R., y Gowen, S. R. (1997). Damage to roots of Musa cultivars by Radopholus similis with and without protection of nematicides. Nematropica, 27(1), 27-32.

- Gowen, S., Quénéhervé, P., y Fogain, R. (2005). Chapter 16: Nematodes parasites of bananas and plantains. En Luc, M., Sikora, J., y J. Bridge (eds.), Plant Parasitic Nematodes in Subtropical and Tropical Agriculture (pp. 611-643). CABI publishing.

- Green, K. R., Afreh-Nuamah, K. (1998). Plantain IPM in Ghana: a case study. En Frison, E. A., Gold, C. S., Karamura, E. B., y Sikora, R. A. (eds.), Mobilizing IPM for Sustainable Banana Production in Africa (pp. 201-208). Proceedings of a Workshop on Banana IPM Held in Nelspruit, South Africa - 23-28 November 1998. INIBAP.

- Grisales, F., y Lescot, T. (1999). Encuesta diagnóstico multifactorial sobre plátano en la zona cafetera central de Colombia. Boletín Técnico No. 18. Feriva S.A. 66p.

- Guerrero, E. (1996). Fundamentos biológicos y estados del arte. En Guerrero, E. (ed.), Micorrizas. Recurso Biológico del Suelo (pp. 181-208). Bogotá, Colombia: Fondo FEN.

- Guzmán P., O. A., Castaño Z., J. (2002). Reconocimiento de Nematodos Fitopatógenos en Plátanos ‘Dominico Hartón' (Musa 
AAB Simmonds), 'Africa', 'FHIA-20' y 'FHIA-21' en Colombia. INFOMUSA, 11(2), 33-36.

- Hauser, S. (2003). Plantain root nematodes: kill them simple. HFC Soil Physics - Agronomy International Institute of Tropical Agriculture. IITA.

- Jenkins, W. R., y Taylor, D. P. (1967). Plant nematology. New York: Reinhold Publ. Corp. 270p.

- Kaplan, D. T. (1994). Molecular characterization of the burrowing nematode sibling species, Radopholus citrophilus and $R$. similis. En Lamberti, F., De Giorgi, C., y McK Bird, D. (eds.), Advances in Molecular Plant Nematology (pp. 77-83). New York: Plenum Press.

- Loos, C. A. (1962). Studies on the life-history and habits of the burrowing nematode, Radopholus similis, the cause of black-head disease of bananas. Proc. Helminthol. Soc. Wash., 29, 43-56.

- Loos, C. A., y Loos, S. (1960a). The blackhead disease of bananas (Musa acuminata). Proc. Helminthol. Soc. Wash., 27, 189-193.

- _ (1960b). Preparing nematode-free banana "seed". Phytopathology, 50, 383-386.

- Luc, M., Sikora, R. A., y Bridge, J. (2005). Plant parasitic nematodes in subtropical and tropical agriculture. 2nd edition. Wallingford, UK: CABI. 871p.

- Maggenti, A., Luc, M., Raski, D., Fortuner, R., y Geraert, E. (1987). A reappraisal of Tylenchina (Nemata). 2. Classification of the suborder Tylenchina (Nemata: Diplogasteria). Revue Nématol, 10(2), 135-142.

- Mai, W., Mullin, P., Lyon, H., y Loeffler, K. (1996). Plant parasitic nematodes. A pictorial key to genera. Fifth edition. Comstock Publishing Associates a Division of Cornell University Press. $277 p$.

- Marín, D. H., Sutton, T. B., y Barker, K. R. (1998). Dissemination of bananas in Latin America and the Caribbean and its relationship to the occurrence of Radopholus similis. Plant Disease, 82(9), 964-974.

- Martínez, H., Pena, Y., y Espinal, C. (2006). La cadena de plátano en Colombia: una mirada global de su estructura y dinámica 1991-2005. En Ministerio de Agricultura y Desarrollo Rural. Documento de trabajo No. 102. Bogotá, Colombia. Obtenido en mayo de 2011, desdehttp://www.agrocadenas.gov.co/platano/documentos/caract erizacion_platano.pdf

- Mbwana, A. S. S., y Rukazambuga, N. D. T. M. (1998). Banana IPM in Tanzania. En Frison, E. A., Gold, C. S., Karamura, E. B., y Sikora, R. A. (eds.), Mobilizing IPM for Sustainable Banana Production in Africa (pp. 237-245). Proceedings of a Workshop on Banana IPM Held in Nelspruit, South Africa - 23-28 November 1998. INIBAP.

- Moens, T., Araya, M., Swennen, R., y De Waele, D. (2004). Enhanced biodegradation of nematicides after repetitive applications and its effect on root and yield parameters in commercial banana plantations. Bio Fertil Soils, 39, 407-414.

- Moens, T., Araya, M., Swennen, R., De Waele, D., y Sandoval, J. (2003). Growing medium, inoculum density, exposure time and pot volume: factors affecting the resistance screening for Radopholus similis in banana (Musa spp.). Nematropica, 33(1), 926.

- Montiel, C., Sosa, L., Medrano, C., y Romero, D. (1997). Nematodos fitoparásitos en plantaciones de plátano (Musa AAB) de la margen izquierda del río Chana. Estado Zulia, Venezuela. Departamento Fitosanitario, Facultad de Agronomía, Universidad de Zulia, Venezuela. pp. 245-251. 
- Nguyet, D., Thuy, T., Tuyet, N., Tu, D., Yen, N., Thanh, D., y Nhi, H. (2003). Occurrence or Pratylechus coffeae and occurrence, damage and reproduction of Radopholus similis en the Northen and Central Highlands of Vietnam. En De la Cruz, F., Van den Bergh, Jr. I., De Waele, D., Hautea, D., y Molina, A. (eds.), Towards Management of Musa Nematodes in Asia and the Pacific (pp. 65-78). Montpellier, France: INIBAP.

- Okech, S. H. O., Karamura, E. B., y Gold, C. S. (1998). Banana IPM in Uganda. En Frison, E. A., Gold, C. S., Karamura, E. B., y Sikora, R. A. (eds.), Mobilizing IPM for Sustainable Banana Production in Africa (pp. 225-236). Proceedings of a Workshop on Banana IPM Held in Nelspruit, South Africa - 23-28 November 1998. INIBAP.

- Oramas Nival, D., y Román, J. (2006). Histopatología de los nematodos Radopholus similis, Pratylenchus coffeae, Rotylenchulus reniformis y Meloidogyne incognita en plátano (Musa acumulata X M. balbisiana, AAB). J. Agric. Univ. P.R., 90(1-2), 83-97.

- Perry, R., y Moens, M. (2006). Plant nematology. London: CAB International. 447p.

- Price, N. (2006). The banana burrowing nematode, Radopholus similis (Cobb) Thorne, in the Lake Victoria region of East Africa: its introduction, spread and impact. Nematology, 8(6), 801-817.

- Quénéhervé, P., Chabrierb, C., Auwerkerkena, A., Toparta, P., Martinya, B., y Marie-Luce, S. (2005). Status of weeds as reservoirs of plant parasitic nematodes in banana fields in Martinique. Crop Protection, 25, 860-867.

- Quénéhervé, P., De Bock, S., Valette, C., y Chabrier, C. (2000). Enzyme phenotypes of Meloidogyne spp. associated with bananas in Martinique (Abstr.). Nematropica, 30, 145.

- Ramírez, C., y Castaño, J. (2009). Efecto del tratamiento químico de la semilla de plátano con diferentes productos y tiempos de exposición sobre la población de nematodos fitoparásitos. Fitotecnia, No. 144. 2p.

- Rivera Alvarado, S., Guzmán Piedrahita, O. A., y Zamorano Montañéz, C. (2011). Arvenses hospedantes de nematodos fitoparásitos en el cultivo de plátano en la granja Montelindo. Trabajo de grado para optar al título de Ingeniero Agrónomo, Universidad de Caldas, Manizales, Colombia.

- Robinson, J. C., Daneel, M., y Schoeman, P. S. (1998). Cultural practices in relation to integrated pest management in bananas. En Frison, E. A., Gold, C. S., Karamura, E. B., y Sikora, R. A. (eds.), Mobilizing IPM for Sustainable Banana Production in Africa (pp. 283-289). Proceedings of a Workshop on Banana IPM Held in Nelspruit, South Africa - 23-28 November 1998. INIBAP.

- Román, J. (1978). Fitonematología tropical. Universidad de Puerto Rico, Est. Exp.

- Sarah, J. L. (2000). Burrowing nematode. En Jones, D. R. (ed.), Diseases of banana, Abacá and Enset (pp. 295-303). Wallingford, UK: CABI Publishing.

- Sarah, J. L., Pinochet, J., y Stanton, J. (1996). El nematodo Barrenador del banano Radopholus similis Cobb. Plagas de Musa. Hoja divulgativa No. 1. Francia: INIBAP.

- Sasser, J. N., y Freckman, D. W. (1987). World perspective on nematology: The role of society. En Veech, J. A., y Dicson, D. W. (eds.), Vistas on Nematology: A commemoration of the Twentyfifth Anniversary of the Society of Nematologist (pp. 7-14). Society of Nematologist, Inc Hyattsville, MD.

- Seshu R., K. V., Ngode, L., Ssenyonga, J. W., Wabule, M., Onyango, M., Adede, T. O., y Ngoze, S. (1998). Management of Pests and Diseases of Banana in Kenya: a status report. En 
Frison, E. A., Gold, C. S., Karamura, E. B., y Sikora, R. A. (eds.), Mobilizing IPM for Sustainable Banana Production in Africa (pp. 215-224). Proceedings of a Workshop on Banana IPM held in Nelspruit, South Africa - 23-28 November 1998. INIBAP.

- Sher, S. A. (1968). Revision of the genus Radopholus Thorne, 1949 (Nematoda: Tylenchoidea). Proc. Helminthol. Soc. Wash., 35, 219-237.

- Siddiqi, M. R. (2000). Tylenchida: Parasites of plants and insects. 2nd edition. CABI Bookshop. 834p.

- Sieverding, E. (1991). Vesicular-arbuscular mycorrhiza management in tropical agrosystems. Eschborn (Alemania), GTZ. 371p. Ing. (Schriftenreihe der GTZ No. 224).

- Smith, S. E., y Read, D. J. (1997). Mycorrhizal symbiosis. 2. ed. San Diego, Estados Unidos: Academic Press. 605p.

- Stover, R. H. (1972). Banana, plantain and abaca diseases. Kew, UK: Commonwealth Mycological Institute. 316p.

- Tarjan, A. C. (1961). Longevity of Radopholus similis (Cobb) in host- free soil. Nematologica, 6, 170-175.

- Thorne, G. (1961). Principles of nematology. New York: McGrawHill. 553p.

- Valette, C., Andary, C., Geiger, J. P., Sarah, J. L., y Nicole, M. (1998). Histochemical and cytochemical investigations of phenols in roots of banana infected by the burrowing nematode Radopholus similis. Phytopathology, 88(11), 1141-1148.

- Wehunt, E. J., Hutchinson, D. J., y Edwards, D. I. (1978). Reactions of banana cultivars to the burrowing nematode. Journal of Nematology, 10, 368-370.

1. M.Sc. Profesor de Nematología, Facultad de Ciencias Agropecuarias, Universidad de Caldas. 